\title{
Multilinguales
}

\section{Les interactions en ligne/ les interactions en présentiel : étude comparative}

Online interactions / face-to-face interactions : a comparative study

\section{Kheira Yahiaoui}

\section{(2) OpenEdition}

\section{Journals}

\section{Édition électronique}

URL : https://journals.openedition.org/multilinguales/4843

DOI : $10.4000 /$ multilinguales.4843

ISSN : 2335-1853

\section{Éditeur}

Université Abderrahmane Mira - Bejaia

\section{Référence électronique}

Kheira Yahiaoui, «Les interactions en ligne/ les interactions en présentiel : étude comparative », Multilinguales [En ligne], 13 | 2020, mis en ligne le 15 juillet 2020, consulté le 18 mai 2021. URL : http:// journals.openedition.org/multilinguales/4843; DOI : https://doi.org/10.4000/multilinguales.4843

Ce document a été généré automatiquement le 18 mai 2021

\section{cc) $(1) \ominus$}

Multilinguales est mise à disposition selon les termes de la Licence Creative Commons Attribution Pas d'Utilisation Commerciale - Pas de Modification 4.0 International 


\title{
Les interactions en ligne/ les interactions en présentiel : étude comparative
}

\author{
Online interactions / face-to-face interactions : a comparative study
}

\author{
Kheira Yahiaoui
}

1 Les changements et l'évolution des sociétés actuelles ainsi que l'ascension technologique ont permis l'émergence poussé à l'émergence de plusieurs moyens de communication permettant aux interlocuteurs d'échanger d'entrer en échange à distance tels que: les chats, les plateformes interactives, les forums, Messenger (Facebook), etc. Ces nouveaux dispositifs d'échanges à distance se présentent comme un autre moyen d'interaction que les locuteurs utilisent et qui se substituent à l'interaction dite face à face ou à l'interaction audio (téléphonique). Dans le même sillage, Kerbrat-Orecchioni (2011: 147) affirme que

l'apparition de ces nouveaux dispositifs de communication nous incite à rebaptiser « en présentiel » (selon un néologisme qui se répand aujourd'hui ; comparaison donc entre le fonctionnement d'une part, du corpus de conversation en présentiel (dorénavant $\mathrm{CP}$ ) et d'autre part, du corpus de conversation en ligne (dorénavant CL).

En français, l'adjectif présentiel est utilisé, depuis le milieu des années 1980, pour désigner une situation de co-présence physique, laissant le terme «face à face » pour décrire toutes les conversations où les participants se font face, que ce soit autour d'une table de café ou via une interface d'écran.

2 Notre travail s'inscrit dans le cadre des recherches qui traitent de l'impact et l'émergence de ces dispositifs en ligne sur les pratiques communicationnelles en tentant de répondre aux questions suivantes : les interactions en ligne : une tendance ou un nouveau genre? Comment se présente le cadre participatif? Et les rituels d'ouverture et de clôture?

Ainsi, l'objectif de cette étude est de démontrer les spécificités de chaque mode et de voir leurs principes fondamentaux sur le plan de la forme et du contenu. S'inscrivant 
dans une approche socio-interactionnelle, notre réflexion se situe dans le cadre de l'analyse des interactions verbales qui nous permettra d'étudier la structure et la forme des deux modes d'interaction et de la sociolinguistique pour mettre le point sur le comportement langagier des interlocuteurs et l'usage de certaines formes relatives aux échanges sur les réseaux sociaux (abréviations, émoticônes, etc.).

\section{Interaction ou conversation : un choix terminologique}

3 L'analyse conversationnelle est une approche qui s'intéresse à l'étude des conversations authentiques. Les premiers travaux dans le domaine sont ceux de Sacks et ses collaborateurs Schegloff et Jefferson qui se sont intéressés à l'analyse des conversations quotidiennes et à leur description en situation naturelle: "les échanges langagiers les plus ordinaires de la vie quotidienne, comme les discours ritualisés, sont des activités socialement structurées que la sociologie peut constituer en objet d'étude. [...] leur intention initiale était de développer une analyse micro-sociologique des échanges discursifs » (Bachmann, Lindenfeld et Simonin, 1991 : 133). La conversation est le centre d'études de la discipline et se présente comme l'architype et le modèle de base de toute situation de communication. Sa multicanalité fait d'elle un domaine très fertile qui peut avoir un apport pour le domaine linguistique et social puisqu'elle est le biais par lequel s'exprime chaque société.

4 Si l'analyse conversationnelle a pour objet l'étude des conversations, les interactions verbales s'intéressent à toutes formes d'échanges : débat, discussion, transaction, etc. Ainsi, on parle d'interaction lorsqu'une action réciproque qui s'établie entre deux ou plus de deux individus. Ainsi, elle « correspond à ce qui se passe lorsque plusieurs personnes se trouvent réunies » selon Traverso (1999: 05). Dans la même réflexion, Vion (2000:17) entend par interaction :

« toute action conjointe, conflictuelle et/ou coopérative, mettant en présence deux ou plus de deux acteurs ».

Autrement dit, l'interaction regroupe toute échange de parole ou d'action en dépit de sa nature et quelle qu'en soit le cadre où elle se déroule.

Pour sa part, Goffman (1973: 23) renvoie l'interaction à l'échange stricte de «face à face » :

«par interaction, on entend l'ensemble de l'interaction qui se produit en une occasion quelconque quand les membres d'un ensemble donnée se trouve en présence continue ${ }^{1}$ les uns des autres; le terme "une rencontre" pouvait aussi convenir ".

Par là, Goffman conditionne l'interaction par une présence «de face à face » des partenaires; ce qui apparaît comme une définition restrictive excluant par là toute autre interaction qui ne se déroule pas selon cette condition de face à face. KerbratOrecchioni (1990) parle d'interaction verbale en réservant ce terme aux activités de communication, ce qui rend le domaine des interactions plus pointues. Elle part du principe que l'interaction est de dimension humaine puisque chaque société s'élabore et évolue à travers l'interactivité.

6 A ce stade, on peut dire que l'interaction présuppose une co-présence en un même lieu d'un certain nombre de personnes entre lesquelles peut s'établir un contact visuel : face à face ou seulement verbal : l'exemple de l'interaction en ligne. De ce fait, nous optons pour l'usage de «interaction» qui nous parait être un terme générique représentant 
tout acte de parole impliquant au moins deux personnes contrairement à " conversation » qui renvoie à cet aspect familier, spontané de l'échange. Il nous faut préciser que notre corpus, regroupe des étudiants qui se connaissent mais dont le degré d'affinité varie d'une personne à une autre. Autrement dit, nous ne pouvons pas déterminer la relation interpersonnelle entre les partenaires de l'échange qui détermine le genre interactionnel mis en place durant ces échanges.

\section{La communication écrite médiatisée par ordinateur (CEMO)}

7 La Communication Écrite Médiatisée par Ordinateur (CEMO) (courrier électronique, forums de discussion. Internet Relay Chat) de sa part est depuis quelques années un moyen de communication très courant au sein des organisations, et elle est parfois considérée comme un outil privilégié de travail collectif et coopératif. Ce mode de communication peut être qualifié de " conversation écrite » ou " interaction en ligne » car il repose sur une utilisation du code écrit dans une dynamique conversationnelle. Marcoccia (2000 : 268) définit la CEMO comme

[...] une forme de communication hybride car elle relève à la fois de la communication écrite et de la conversation. Le code utilisé est l'écrit, mais les échanges de messages entrent dans une dynamique conversationnelle, essentiellement à cause de la rapidité de la rédaction et de la transmission des messages.

De ce fait, les messages médiatisés par ordinateur sont marqués par l'oralité et se conçoit comme une forme hybride entre l'écrit et l'oral.

La notion d'interaction a toujours suscité l'intérêt des linguistes notamment en analyse conversationnelle, néanmoins, l'interaction en ligne qui a constitué un champ fertile de recherche ces dernière années et poussant à l'émergence d'un nouveau terrain de recherche sur les pratiques et le discours numérique. Paveau (2017:14) précise que :

«le discours s'est technologisé et nos modes d'écriture et de lecture portent les traces de cette conversion numérique du langage $»$.

Pour elle le discours numérique recouvre des situations technodiscursives différentes, ainsi, elle propose une typologie à trois entrées qui repose sur des critères linguistiques: numérisé, numérique, numériqué. Cette répartition correspond entièrement à celle que Dacos et Mounier (2010:6-7) proposent lorsqu'ils soulignent : qu'il s'agit d'un domaine rassemblant trois pratiques différentes : la numérisation comme «portage du texte imprimé », l'édition numérique qui exploite « nativement les possibilités qu'offre le texte numérique » et l'édition en réseau qui développe « de nouveaux modes de production des textes.

\section{L'interaction en ligne (il) : postulat et définition}

9 Les chercheurs en linguistique, en sociologie et en psychologie ont commencé à s'intéresser récemment à ce qui a été très vite perçu comme une révolution majeure de la communication. Les développements de l'informatique et des télécommunications ont fourni, à un public de plus en plus large, de nouveaux outils de communication. Les chercheurs anglophones furent les précurseurs dans l'étude des échanges en ligne en créant le terme Computer Mediated Communication (CMC) (Herring, 1996) pour désigner ces nouvelles formes d'interaction à l'aide de l'outil numérique. Ce terme a été traduit 
en français par communication médiatisée par ordinateur (Mondada, 1999). On préfère l'usage de communication médiée par ordinateur qui semble plus adéquat dans ce genre de situation d'échange indiquant le moyen ou le médium via lequel passe l'échange à l'instar de médiatiser qui stipule que la communication existait avant et elle fut découverte par l'invention de l'ordinateur.

Contrairement aux autres moyens de communication numérique tels que les courriers électroniques et bien d'autres, l'échange par Messenger a une visée communicative qui se concentre sur les aspects sociaux-relationnels. En d'autres termes, ce moyen est rarement utilisé pour des fins professionnelles. Cependant, nous ne pouvons pas affirmer ce postulat vu que la relation interpersonnelle des interlocuteurs ne nous positionne pas dans un échange relatif à une conversation, ce qui justifie notre choix de l'usage du terme interaction.

Cette nouvelle tendance de communication devient virale avec l'ouverture d'internet et la création des espaces d'échanges numériques faisant d'elle une partie du quotidien des interlocuteurs qui n'ont besoin que d'un smartphone et d'une application pour entrer en interaction avec leur entourage. Ainsi, l'appellation Communication médiée par ordinateur se trouve en décalage avec la réalité de cet échange. Donc, nous optons pour la terminologie interaction en ligne (IL) pour caractériser cette situation de communication qui reprend les principes de base de la communication quotidienne en l'adoptant dans une forme numérique. Nous jugeons que cette forme est un autre moyen d'interaction où Internet devient indispensable dans le quotidien des locuteurs.

\section{Corpus et méthodologie}

11 Les méthodes de travail en analyse des interactions sont sensiblement différentes de celles utilisées en linguistique pour étudier le système de la langue. La démarche adoptée part des données et cherche à identifier des comportements interactionnels récurrents. A partir de ces observations, elle propose des catégorisations et des généralisations en adoptant une démarche descriptive et analytique. L'étude repose sur un corpus authentique enregistré au moment de sa production et transcrit selon des conventions propres à l'oral. Dans le cas de notre étude, nous regroupons deux corpus d'étude : un premier corpus d'enregistrement audio d'interactions entre les étudiants durant la journée à l'université, un second corpus relatif à leur interaction sur Messenger durant la journée ou la soirée. En 2019, du mois d'avril et jusqu'à la fin du mois de juin, a eu lieu notre travail d'enquête et de collecte auprès des étudiants de master II « linguistique » de l'université Oran 2. L'enquête a été faite en notre présence après avoir expliqué à nos informateurs, l'objet et l'objectif de ce travail.

Nous travaillons actuellement avec un groupe d'étudiants constitué de 09 filles et de 03 garçons inscrits en filière de français et qui se répartissent comme suit :

Tableau 01

\begin{tabular}{|l|l|l|l|}
\hline & $3^{\text {ème }}$ année licence & $1^{\text {ère }}$ année master & $2^{\text {ème }}$ année master \\
\hline Filles & 01 & 05 & 03 \\
\hline Garçons & $/$ & 02 & 01 \\
\hline
\end{tabular}


Nous avons obtenu le corpus ${ }^{2}$ suivant :

Tableau 02

\begin{tabular}{|l|l|l|}
\hline Nombre des jeunes (élèves) & Interaction en présentiel & Interaction en ligne \\
\hline Dilogue fille & 06 & 15 \\
\hline Dilogue mixte (F/G) & 04 & 07 \\
\hline Polylogue fille & 08 & 12 \\
\hline Polylogue mixte (F/G) & 13 & 04 \\
\hline
\end{tabular}

13 Pour les interactions en présentiel, notre corpus est composé d'enregistrements audio des interactions entre étudiants que nous avons recueillis et transcrits. Pour les interactions en ligne, les étudiants nous ont remis des captures d'écran de leurs interactions via Messenger. C'est sur la base d'une approche séquentielle que nous proposons une analyse du contenu et de la structure de ces interactions. En d'autres termes, nous analysons, dans ces deux corpus, de manière aussi exhaustive que possible, leurs aspects linguistique et interactif.

\section{Les interactions via messenger : un terrain pas comme les autres!}

Internet est un espace social où se croisent et coexistent divers espaces sociaux, Comme dans d'autres terrain de recherche, le chercheur doit donc commencer par découvrir le terrain, l'apprivoiser et le décrire comme le précise Héas et Poutrain (2003:4) :

Cet espace virtuel se présente au chercheur tel une ville qu'il ne connaît pas et qu'il va apprendre à découvrir. Par tâtonnements et une présence prolongée sur la toile, l'anthropologue se familiarise avec les réseaux qui s'y trouvent. [...] L'anthropologue se retrouve face à un espace qu'il lui faut décrire, où il doit prendre ses repères. Il évalue ainsi les ressources dont il dispose.

En Algérie, Facebook compte désormais près de 15 millions $^{3}$ de comptes utilisateurs. Ce réseau généraliste est redoutable, tellement redoutable que d'autres réseaux sociaux affichent désormais des statistiques en baisse (en matière de popularité).

Du coté social, l'Algérien est de plus en plus présent sur Facebook car ce réseau lui offre l'opportunité d'approcher l'autre, de discuter et de créer des liens amicaux. Nous présentons dans ce qui suit les statistiques ${ }^{4}$ relatives à l'usage de ce réseau en Algérie par région géographique et par sexe. 
Chiffres Clés de Facebook en Algérie Top wilayas nombre d'utilisateurs Facebook

Nombre d'utilisateurs Facebook

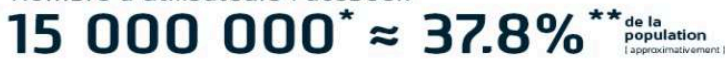
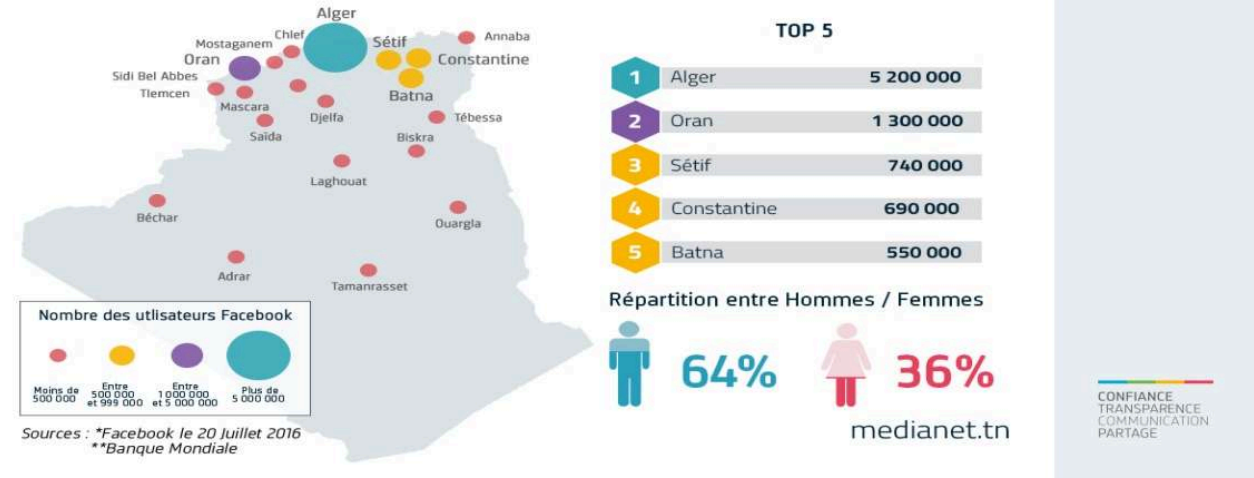

Par ailleurs, une étude établie en 2017 a démontré que toutes les tranches d'âge - à pourcentage différent - s'intéressent à ce réseau. Ainsi, plus de $67 \%$ de la population algérienne présente sur Facebook est âgée de 18 et 35 ans ; $15 \%$ concernent les moins de 18 ans et $18 \%$ les plus de 35 ans. Les résultats chiffrés de cette étude nous amène à dire qu'il s'agit d'un réseau plus ou moins homogène et jeune comme l'indiquent les chiffres suivants ${ }^{5}$ :

\section{MEDIANET}

\section{Chiffres Clés de Facebook en Algérie Répartition par tranche d'âges}

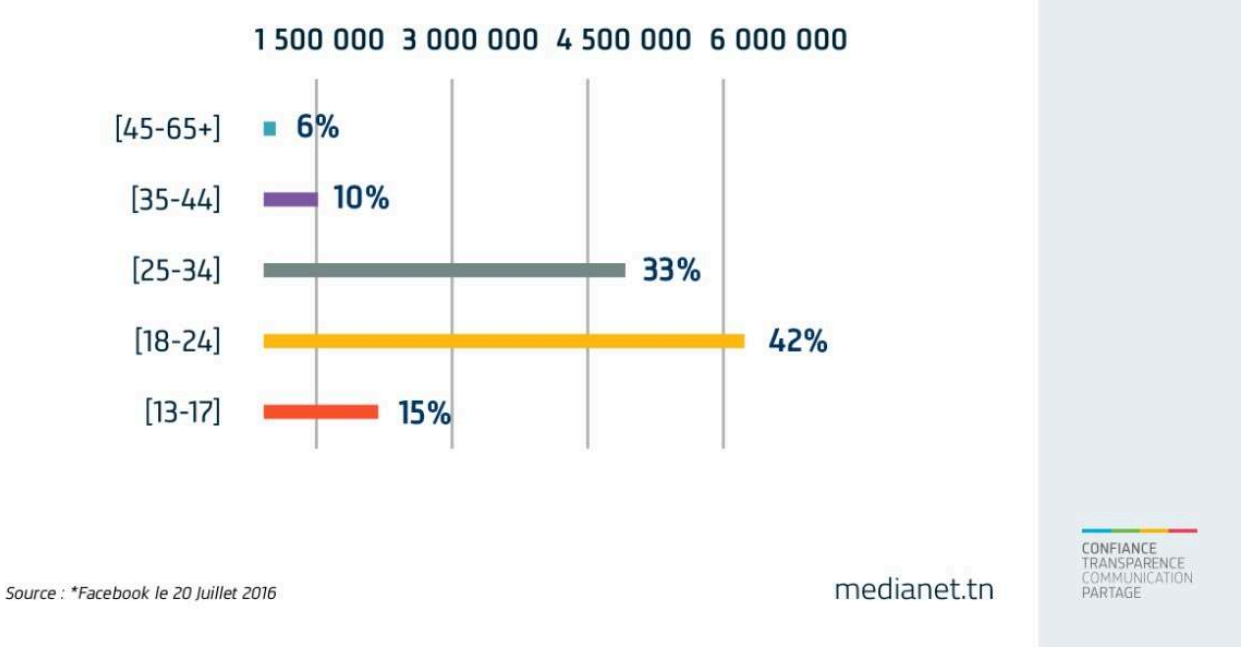

Ainsi, nous avons choisi de mener notre enquête interactionnelle à travers ce réseau vu le nombre de connectivité de la population surtout que ces dernières années nous assistons à la version " light ${ }^{6}$ » de ce réseau en Algérie et à travers le monde. A l'instar des précédents travaux sur les interactions en ligne notamment celui KerbratOrecchioni (2011) qui se penche sur la question à travers un échange audio-visuel à travers une plateforme d'échange. Dans la même lignée et s'inscrivant dans la même démarche comparative, notre travail tente de relever les spécifiés d'un échange 
distanciel via Messenger où les usagers disposent du moyen écrits et d'autres supports. En effet, la temporalité des échanges ne correspond pas à celle d'une interaction face à face où les participants ont un temps de réflexion avant de répondre et le recours à l'écrit induit quasiment une temporisation (corriger des fautes de frappe par exemple, reformuler le message, utiliser les émoticônes, etc.). Par ailleurs, cette forme d'échange peut aussi laisser place à des malentendus, superposition de discours, désordre thématique, etc.

Il est important de signaler que l'un des avantages que présente une étude des échanges en ligne est la possibilité d'archivage des échanges ce qui permet au chercheur de revenir à tout moment pour des fins d'analyse. Notons, que nous n'avons pas pris en charge les appels et « les mémos vocaux ${ }^{7}$ » disponibles sur ce réseau.

\section{Présentation de la situation de communication : interaction en présentiel (IP) vs interaction en ligne (IL)}

L'approche interactionniste se caractérise par le recours à des énoncés actualisés dans des situations communicatives particulières. La situation de communication constitue un élément central de l'analyse des interactions verbales. C'est en effet les éléments du contexte qui permettent de déterminer le mode d'interaction observée. Décrire la situation de communication nous permettra par la suite de cerner les spécificités d'une $\mathrm{IP}^{8}$ par rapport à une $\mathrm{IL}^{9}$. Les différents éléments constitutifs de toute interaction nous servent à identifier son mode afin de mieux l'analyser. Il est donc clair qu'on ne peut pas faire abstraction de la situation de communication qui détermine le fonctionnement et l'organisation des interactions verbales. Les éléments contextuels sont à intégrer dans la description des échanges, car ils permettent d'expliquer certains phénomènes insaisissables en dehors de la situation. D'où la nécessité de décrire et d'observer les données in situ c'est-à-dire au sein de leur environnement contextuel.

\section{Setting ou cadre spatio-temporel}

L'IP se déroule dans un cadre public (dans les couloirs de l'université, la cafétéria ou la cour). Les interactions sont synchrones et linéaires dans le temps. En d'autres termes, on observe les ratés, les chevauchements, les interruptions, les rires, etc. Elles sont multicanales et multimodales.

Séquence :

$\mathrm{C}_{23}$ : Oui anā : je travaille sur les interférences $\mathrm{f}$ le premier chapitre deret des définitions $\mathrm{w} \underline{\text { [fdeuxième }}$

$\mathrm{A}_{78}$ : [rākī dā :yerā les interférences en écrit (tu as fait)

Dans cet extrait, nous observons un chevauchement suite à une superposition de deux tours de paroles où ce phénomène est très récurrent et relatif aux interactions orales de même que les pauses qui se présentent par ( : ) indiquant une pause brève.

Dans les interactions en ligne, le locuteur passe par un dispositif qu'il peut utiliser dans un lieu public ou privé pour échanger. De même, il pourra être en échange avec 
plusieurs personnes même temps ${ }^{10}$.

Elles ne dépendent pas des modifications spatio-temporelles des interlocuteurs qui sont en lien constant via l'application Messenger sur leurs Smartphones. Ainsi, les interlocuteurs adoptent une posture virtuelle de réciprocité leur permettant d'être en présence dans différentes situations publiques ou privées. Autrement dit, nous déduisons une certaine stabilité du cadre sauf dans certains cas de figure

. A contrario, les interactions face à face sont conditionnées par la situation de communication qui pèse sur son déroulement impliquant ainsi " une relation systématique entre arrangement spatial et mode d'interaction "

(Kendon, 1992 : 330).

\section{Participants}

Les participants sont en présence continuelle tout au long de l'interaction comme le souligne Kerbrat-Orecchioni (1990 : 216)

" pour qu'en ai affaire à une seule et même interaction, il faut et suffit que l'on ait un groupe de participant modifiable mais sans rupture, qui dans un cadre spatiotemporel modifiable mais sans rupture, parlant d'un objet modifiable mais sans rupture ».

L'interaction est conditionnée par "la continuité» (sans rupture: de thèmes, des groupes...), puisque le terme "modifiable" risque d'être pris comme un indice de clôture. Il est à noter que dans certains cas le cadre participatif se voit modifié avec l'ajout ou le retrait d'un participant cependant le critère de la continuité est toujours maintenu.

La spécificité de l'interaction en ligne est que les participants sont reconnaissables par des pseudonymes dans les échanges ${ }^{12}$. De même, l'interaction n'aura lieu que si l'un des participations écrit un message pour lancer l'ouverture. Il peut être connecté sans entrer en interaction avec son partenaire ou avec le groupe d'échange. La présence des participants est visible par une lumière verte (indiquant que la personne est en ligne) qui s'affiche en haut du profil comme l'indique l'image suivante.

Figure 01

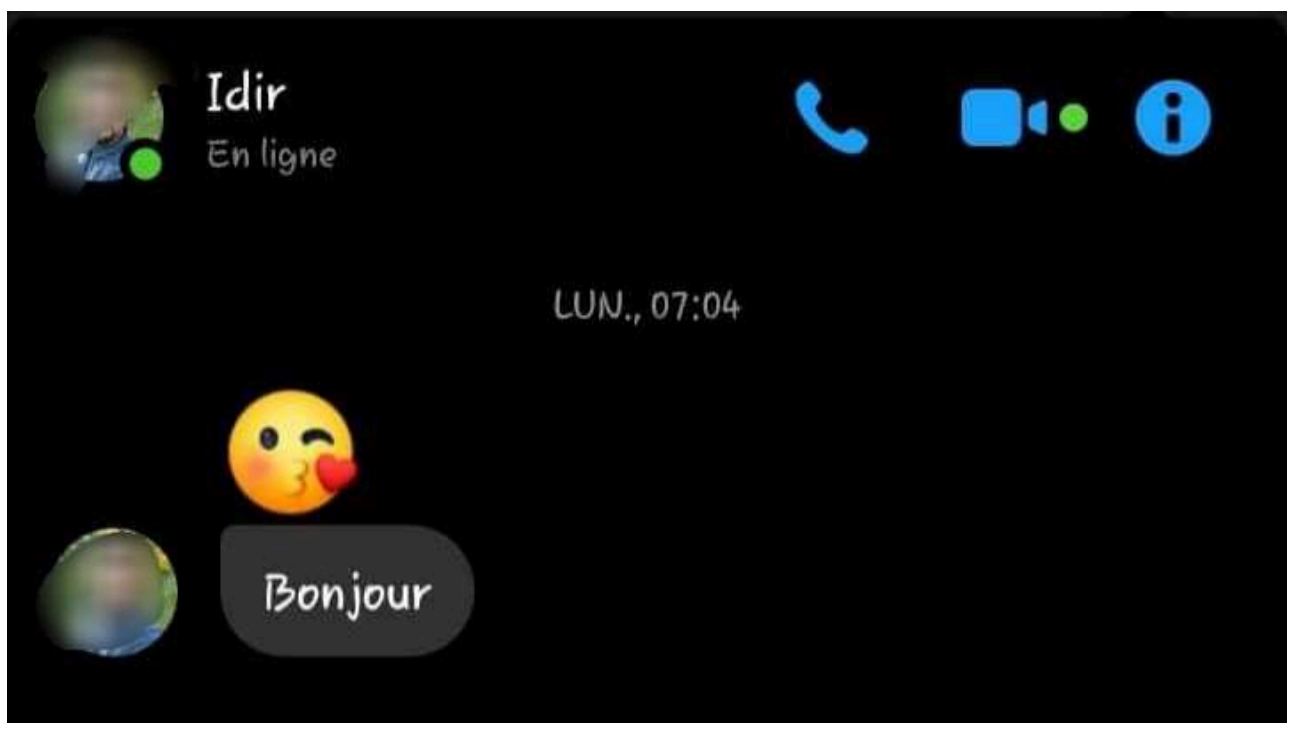


23 position d'interlocuteur sans réagir (mais une bulle avec l'image de la personne indique
que le message est lu ou vu) ou s'impliquer en laissant une trace écrite indiquant son
implication dans l'échange. Cependant, dans les deux cas de figure, on considère que le
participant est ratifié dans les interactions en ligne. De ce fait, de nombreux problèmes
dans les interactions en présentiel sont évités. En d'autres termes, les participants dans
les IL sont automatiquement ratifiés, contrairement à IP où on peut avoir des
"passants » ou des « spectacteurs» (bystanders) non ratifiés. On conclut que les IL se
présentent dans un cadre simplifié par rapport à IP.

\section{La finalité}

Elle pourra être interne ou externe selon le genre interactionnel dont il est question. Le corpus de notre étude, réunit des interactions entre amis ayant une finalité interne et d'autres entre étudiants de classe ayant parfois une finalité externe (demande à propos des cours, des travaux de recherche, etc.)

La relation interpersonnelle et la thématique dans ce mode définit la finalité de l'interaction. Dans les polylogues de notre corpus, il s'agit plus d'une finalité externe (pour demander des nouvelles de chacun, pour échanger des informations, etc.). Le reste des dilogues réunit des interactions entre amis ayant une finalité interne comme dans l'exemple suivant :

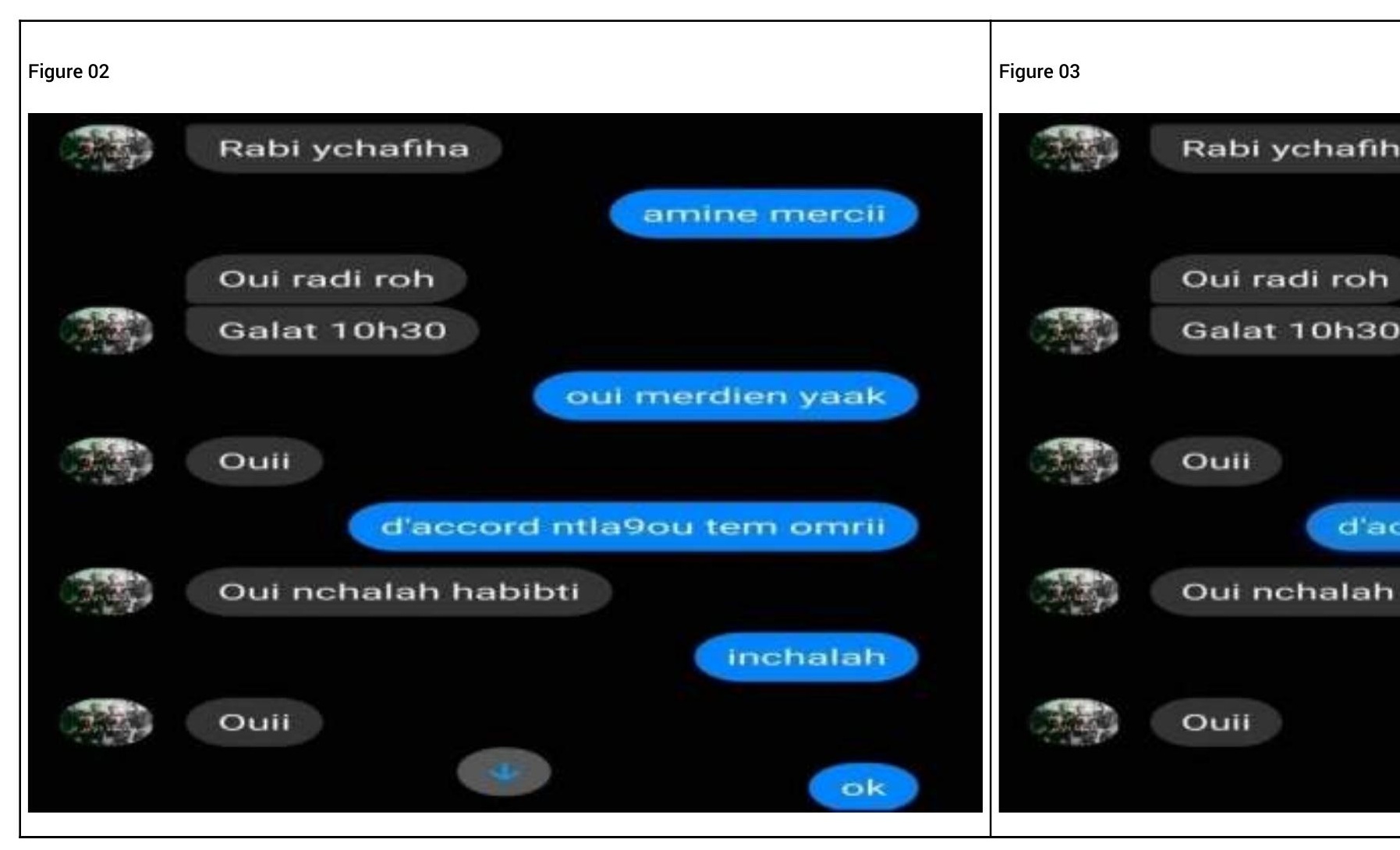




\section{Le contenu de l'échange}

La question sur la finalité nous mène à aborder la forme des interactions en essayant de voir le contenu de leur réalisation: est-ce qu'il s'agit d'interactions formelles ou informelles? Les interactions en présentiel, vu qu'elles sont orales, se présentent sous un modèle plus ou moins formel où nous remarquons certains écarts par rapport à la norme grammaticale comme dans l'exemple suivant :

Séquence :

$\mathrm{B}_{176}:$ Je me rappelle pas son nom Hadā :k lprofesseur yeeayetū :lū des étrangers des français pires des canadiens yegū :lū expliqenā les mots yaeetĩ :leHūm des informations très importantes

Et d'autres écarts par rapport à la langue où nous avons enregistré la présence d'une alternance codique et d'une pluralité linguistique dans la réalisation de ces échanges entres les interlocuteurs à l'instar de l'exemple suivant :

Séquence :

$\mathrm{A}_{90}$ : Le plan voi : :la les titres li hadirīnūm $\mathrm{f}$ le plan ša kūnet rāyehā nedīr finalement xerejelī ga : : $\varepsilon((.)$.$) criquitāwahlī :$

Quant aux interactions en ligne, nous avons relevé un fonctionnement différent :

Dans les dialogues, on a plus une réalisation informelle : écart par rapport aux règles, alternance codique, présence des émoticônes, phrases incomplètes, etc. dont voici quelques extraits :

Figure 04 Figure 05 Figure 06 

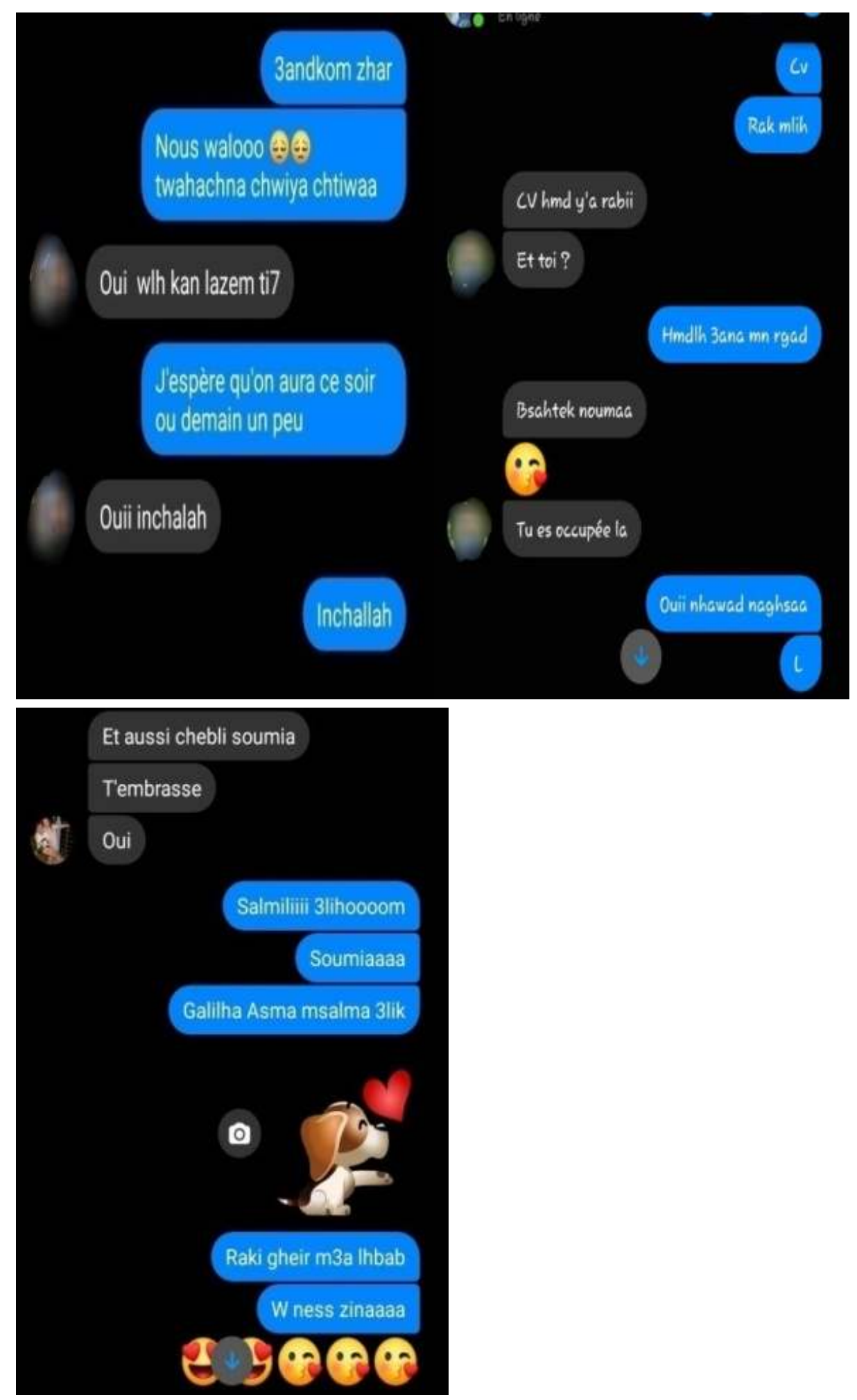

29 A travers ces points relatifs à la situation de communication, nous pouvons conclure que le mode de fonctionnement des interactions est différent. Les interactions en présentiel offrent la possibilité d'être en présence physique et/ou psychologique lors de leur déroulement, elles sont linéaires et se déroulent dans le temps offrant au chercheur une panoplie de phénomènes (relatifs à l'oral) à analyser. Tandis que les interactions en ligne reposent sur un dispositif pour leur déroulement, les partenaires 
ne sont pas en présence l'un à l'autre, recours aux émoticônes pour reprendre certains traits de l'oral (rire, sentiment, etc.).

\section{Le fonctionnement des rituels dans les deux modes : IP et IL}

\section{La séquence d'ouverture}

La fonction principale de la séquence d'ouverture est la mise en place de conditions favorables à l'interaction, conditions aussi bien physiques (bonne distance, bonne installation, etc.) que psychologiques (Yahiaoui, 2010) (reconnaissance mutuelle, acceptation de l'interlocuteur, etc.). Le rituel d'ouverture selon Bange (1992:212) est une

« séquence stéréotypée dont la fonction est d'assurer la mise en route coordonnée

des actions de communication au plan formel».

31 Ainsi, elle consiste à commencer et/ ou à entrer en interaction, c'est une prise de contact avec l'autre partenaire de l'échange ( $C f$. Yahiaoui, 2016). La séquence d'ouverture comporte deux éléments essentiels :

- -Les salutations qui sont un acte de reconnaissance et un rituel d'accès (Goffman, 1974). Elles se réalisent verbalement (bonjour, salut, bonsoir, etc. ), non verbalement ou à l'aide des expressifs (sourire, etc.).

- -Les salutations complémentaires qui comportent des questions de santé, d'humeur, de famille, etc. La plus récurrente d'usage est « comment ça va?».

32 Nous avons dans l'exemple suivant une interaction polylogue où la séquence d'ouverture se présente comme suit:

Séquence :

(T1) $\mathrm{A}_{1}$ : Nadia bonjour' ( :) Nadia

(T2) $\mathrm{B}_{2}$ : oui' bonjour Mounia les salutations proprement dites

(T4) $\mathrm{A}_{3}$ : wech, çava : :

(T5) $\mathrm{B}_{4}:$ euh//très bien' les salutations complémentaires

(T6) $C_{5}:[<+$ ça va :: '

Cet extrait nous renseigne sur la présence des deux composantes de la séquence d'ouverture. Ainsi, les locuteurs, en général, accordent une intention particulière à l'installation de l'ouverture dont le rôle est capital pour la suite de l'échange. La majorité de notre corpus, comporte les deux éléments avec des variations au niveau de la réalisation (question sur l'humeur, la santé, etc.)

Dans les interactions en ligne, l'ouverture est aussi importante au point que, dans les salutations de groupe, les salutations sont souvent accompagnées de l'identifiant de la personne comme dans l'exemple suivant : 
Figure 07

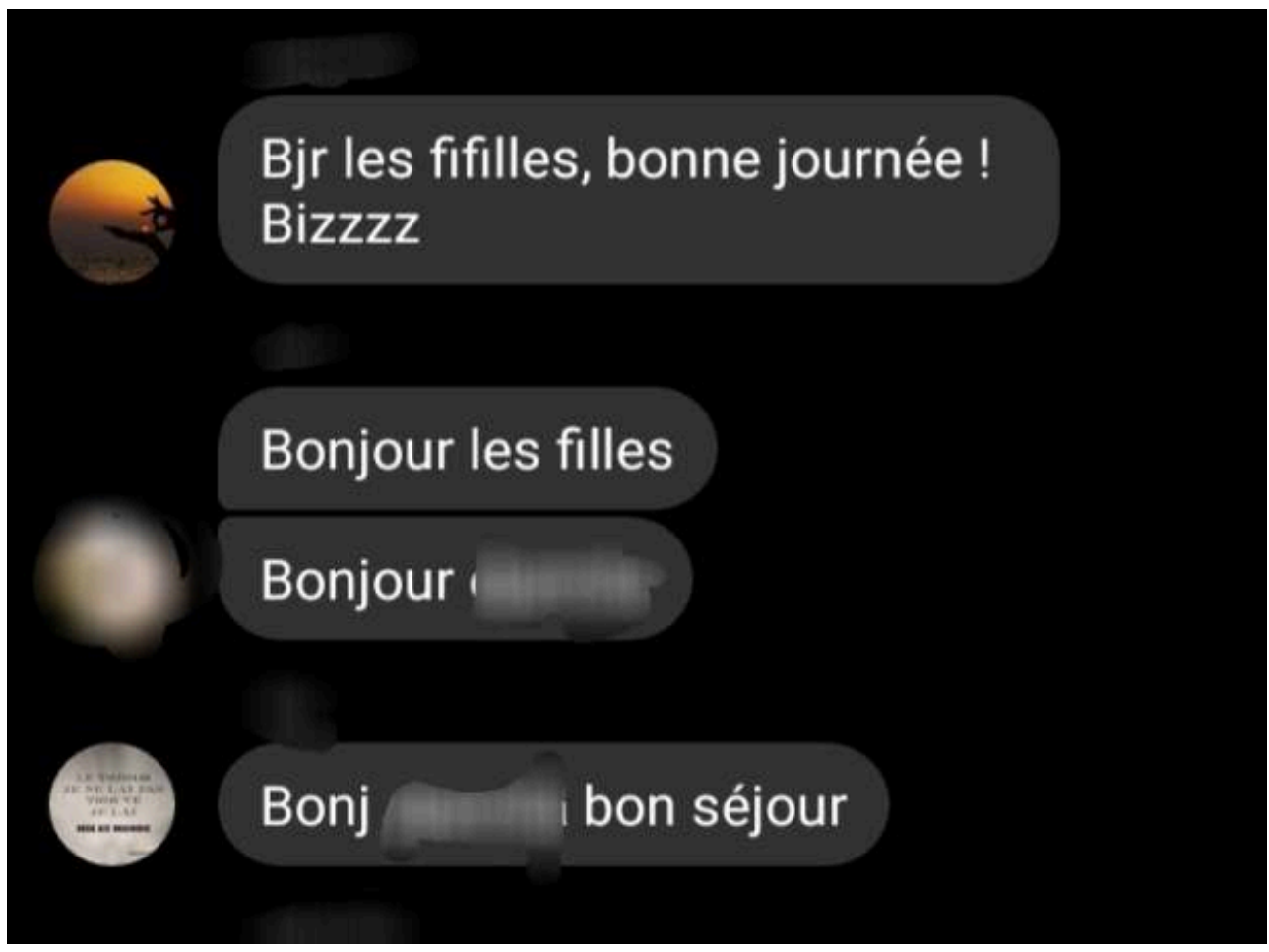

Cette association des salutations avec l'identifiant ${ }^{13}$ est très présente dans les interactions différées et dans les espaces virtuels comme dans le cas de notre étude. Notons aussi la présence des salutations complémentaires où les locuteurs demandent des nouvelles des uns et des autres : 


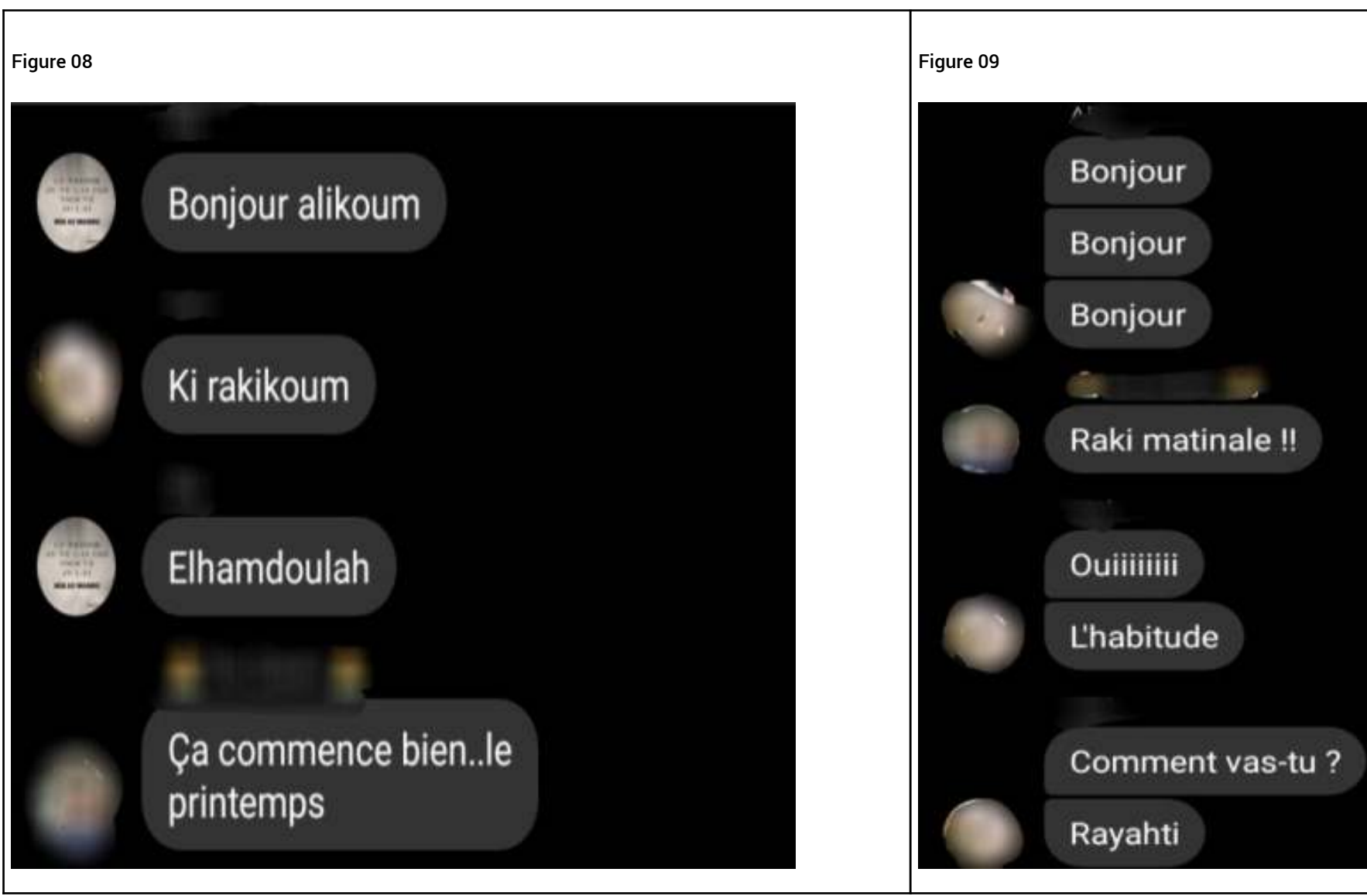

Dans les deux extraits, nous avons les salutations proprement dites suivies des salutations complémentaires qui, dans le premier exemple, préparent l'installation du corps et, dans le second, tendent à avoir des informations sur le partenaire de l'échange.

\section{La séquence de clôture}

La clôture est une étape où les participants s'apprêtent à finir l'échange. Comme l'indique Traverso (1999:32) :

« la clôture correspond à la fermeture de communication et à la séparation des participants ».

En effet, l'ouverture et la clôture sont des séquences qui encadrent l'interaction: l'ouverture a pour fonction d'ouvrir une interaction tandis que la clôture l'oriente vers sa fermeture. La séquence de clôture comporte : la pré-clôture et la clôture proprement dite.

Dans les interactions en présentiel, nous avons repéré la présence d'au moins une composante de la séquence de clôture et parfois les deux ensembles comme dans les exemples suivants :

Séquence :

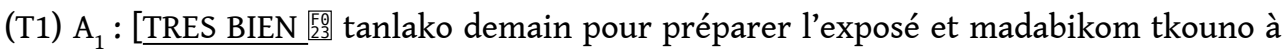
l'heure rire)

(T2) $\mathrm{C}_{2}$ : OK merci : : : : bonne journée 
Dans cet extrait, nous avons la réalisation de séquences de pré-clôture sans pour autant passer à la clôture proprement dite. L'interaction s'achève avec les vœux et des rendezvous de rencontre.

Séquence :

(T1) $A_{1}$ : BON jma3a j'ai un cours dorka, ciao 23

(T2) $B_{2}$ : on se voit après ton cours

(T3) $\mathrm{A}_{3}$ : NON $\left[\begin{array}{l}\text { [ } \\ 33\end{array}\right.$ machakitch , 3andi entrainement

Nous avons, dans cette séquence, la mise en place de la clôture en (T1) qui est négocié en (T2) par son partenaire. En (T3), le locuteur A met fin à la négociation et finit l'interaction.

Dans le cadre des interactions en ligne, les salutations de clôture sont rarement réalisées vu que les locuteurs peuvent à n'importe quel moment reprendre le fil de l'interaction. Cependant, nous avons noté la présence de vœux relatifs à la pré-clôture :

Figure 10

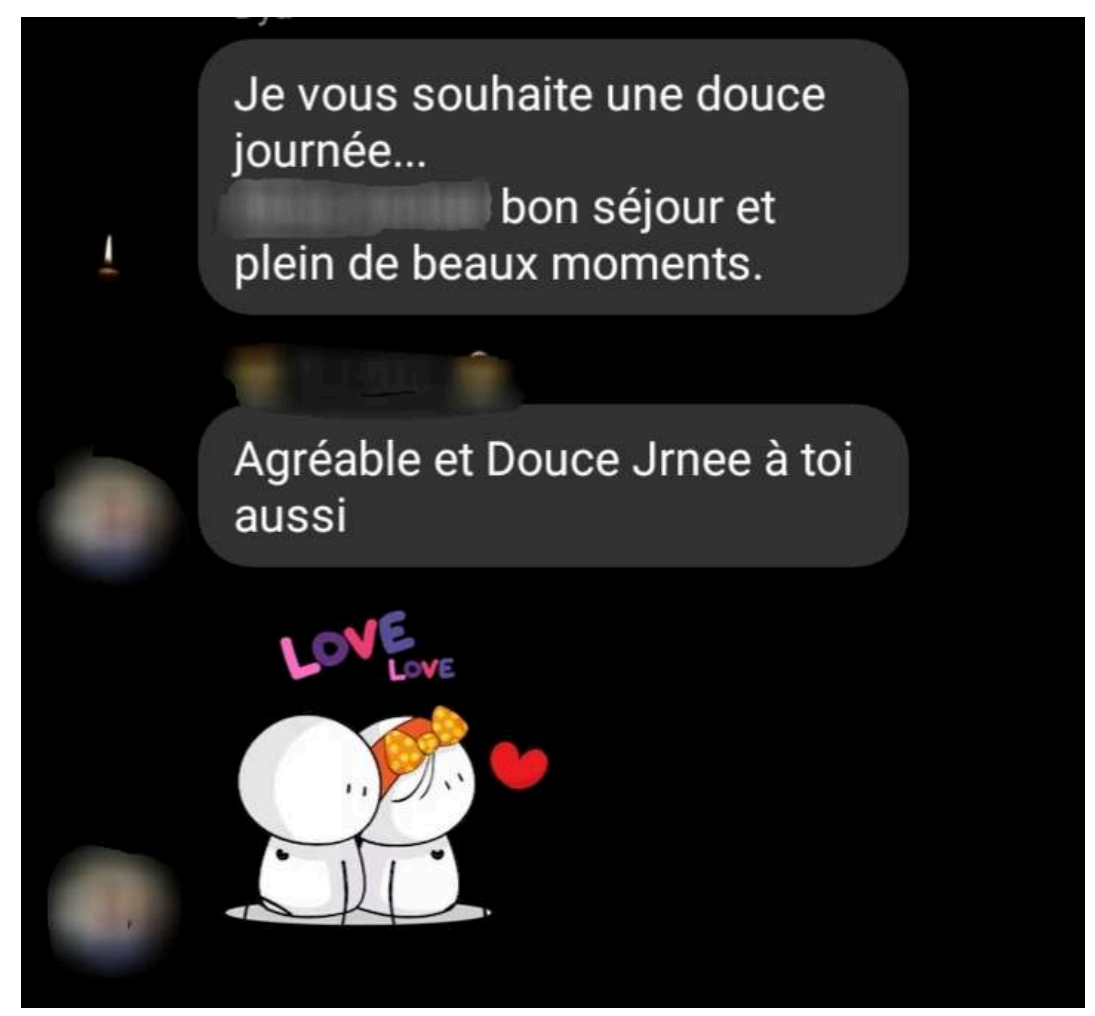

Dans cet extrait, nous avons une interaction polylogue où les interlocuteurs procèdent à des vœux aux membres du groupe sans passer à l'étape de la clôture proprement dite, ce qui fait la spécificité de ce genre d'échange en ligne.

Après avoir présenté quelques différences entre l'interaction en présentiel et en ligne, nous ne pouvons que conclure à l'importance qu'occupe « l'interaction » dans l'analyse conversationnelle et à l'intérêt des auteurs-chercheurs de construire un cadre d'analyse et d'étude sur les échanges réels et virtuels. Ainsi, l'interaction en ligne se présente comme la nouvelle tendance d'échange avec l'émergence et la multiplicité des réseaux sociaux en Algérie assurant une connectivité et offrant des multiples formes d'interaction en un simple clic. Les rituels d'ouverture et de clôture, constituant les moments les plus délicats dans l'interaction en présentiel, se résument dans 
l'interaction en ligne à des émoticônes et des messages brefs de salutations entre les partenaires qui répondent tardivement aux messages affectant ainsi l'organisation des échanges et la progression thématique.

En effet, l'objectif de cette étude comparative est d'expliquer le recours à l'utilisation d'Internet pour créer ou renforcer les liens sociaux. Ce dispositif multiplie les interactions et affaiblit les cloisonnements sociaux qui marquent traditionnellement la sociabilité. Avec ce média, un certain nombre de contraintes traditionnelles de la communication sembleraient levées $d u$ moment où les notions d'affordance communicative sont inclues dans l'interaction en ligne, une théorie du discours qui prend en compte la sémiosis, les participants vivent dans ces espaces virtuels à travers leurs actes.

En outre, les différences interindividuelles ne sont pas bannies et on assiste à des phénomènes de "présence à distance", ce sentiment de proximité avec son interlocuteur, même à distance, permis par les moyens de communication. Donc, il ne faut pas se focaliser uniquement sur l'échange en ligne mais sur la façon dont un échange est accompli en tant qu'interaction technologiquement située.

Ces résultats empiriques ne sont que le début d'une étude interactionnelle qui nécessite de voir d'autres aspects dans l'analyse à l'instar la politesse en ligne, la structure des échanges, les formes orales (mémos vocaux) ainsi qu'une analyse des topics lors de ces échanges. De plus, le corpus doit comprendre d'autres informateurs en vue de questionner les pratiques langagières en ligne et aller vers une étude sur l'identité numérique dans ces interactions.

\section{BIBLIOGRAPHIE}

BACHMANN, C., LINDENFELD, J., SIMONIN, J

BACHMANN, C., LINDENFELD, J., SIMONIN, J

., Langage et communications sociales, Paris, Hatier/Crédif, Coll. LAL. 1991. 223p.

BANGE, P., Analyse conversationnelle et théorie de l'action, Paris, Hatier/Didier, Coll. LAL. 1992. 233p.

DACOS, M., MOUNIER, P., L'Édition électronique, Paris, La Découverte, 2010.

DEVELOTTE, C., KERN, Richard., LAMY, Marie-Noëlle., (Dir.). Décrire la conversation en ligne. Le face à face distanciel, ENS Editions. 2011. 245p.

GOFFMAN, E., La mise en scène de la vie quotidienne, 1.la présentation de soi, Paris, Minuit, Coll. Le sens commun, 1973, 256p.

HEAS, S., et POUTRAIN, V., « les méthodes d'enquête qualitative sur internet », ethnographiques.org [en ligne], no 4, 2003, [consulté le 06/06/17].

HERRING, S. “Computer Mediated Communication”, Linguistic, social and cross-cultural perspectives. Amsterdam / Philadelphia: John Benjamins, 1996. p. 1-10. 
KENDON, A., "The negociation of context in face-to-face interaction". In A. Duranti et C. Goodwin (éds.), Rethinking context. Language as an interactive phenomenon. Cambridge : Cambridge University Press, 1992. p. 323-334.

KERBRAT-ORECCHIONI, C., « Conversation en présentiel et conversation en ligne : bilan comparatif ", Décrire la conversation en ligne. Le face à face distanciel, Develotte , C. et Alii. , (Dir.), ENS Editions, 2011. p. 137-195.

KERBRAT-ORECCHIONI, C., Les interactions verbales, T.2, Paris, Armand Colin, 1992. 386p.

KERBRAT-ORECCHIONI, C., Les interactions verbales, T.1, Paris, Armand Colin. 1990. 347p.

MARCOCCIA, M., « La représentation du non verbal dans la communication écrite médiatisée par ordinateur ", In, journals open edition, $\mathrm{N}^{\circ} 18,2000$, p. 265-274, URL : https://

journals.openedition.org/communicationorganisation/2431. [Consulté le 26/09/2019 à 9h :11].

MONDADA, L., « Formes de séquentialité dans les courriels et les forums de discussion. Une approche conversationnelle de l'interaction sur Internet. ", Alsic [En ligne], vol. 2, n 1 | 1999, URL : http://journals.openedition.org/alsic/1571[Consulté le 20/08/2019].

PAVEAU, Marie-Anne. «L'analyse du discours numérique. Dictionnaire des formes et des pratiques ", Paris, Hermann, 2017, 400p.

TRAVERSO, V., L'analyse des conversations, Paris, Nathann 1990, 128p.

VION, Robert. La communication verbale. Analyse des interactions, Paris, Hachette, 2000. 302p.

YAHIAOUI, K., «L'analyse des salutations dans les interactions entre animateur - auditeur intervenant. Le cas des émissions radiophoniques de divertissement d'Alger Chaîne 3 ", in, Signes, Discours et Sociétés 6, Discours et institutions [en ligne], 23 décembre 2010. Disponible sur Internet : http://revue-signes.info/document.php?id=2222

YAHIAOUI, K., « Les usages linguistiques dans les émissions de divertissement radiophoniques d'Alger Chaîne III ", Questions de communication, $N^{\circ}$ 20, Bonhomme, M. et alii, Nancy, PUN, 2016. p. 49-63.

\section{NOTES}

1. : Nous soulignons.

2. : Les chiffres ne reflètent pas le corpus global de l'étude, il s'agit d'un échantillon sélectionné pour le présent article.

3. : http://blog.medianet.com.tn/blog/chiffres-cles-de-facebook-en-algerie-0 (consulté le 05-05-2020).

4. : http://blog.medianet.com.tn/blog/chiffres-cles-de-facebook-en-algerie-0 (consulté le 05-05-2020).

5. : http://blog.medianet.com.tn/blog/chiffres-cles-de-facebook-en-algerie-0 (consulté le 05-05-2020).

6. : Messenger de Facebook «Light » est la dernière nouveauté pour assurer une connectivité permanente en rupture de solde où Les internautes peuvent accéder au contenu écrit sur ce réseau et recevoir mes messages.

7. : Nous laissons cet aspect de l'étude pour de futurs travaux sur la question de ce genre d'échange dans ce dispositif en ligne.

8. : IP : interaction en présentiel

9. : IL : interaction en ligne 
10. : Les interactions sur Messenger offre la possibilité de communiquer avec plusieurs personnes en même temps, cependant, on ne pourra parler de trilogue ou polylogue sauf dans le cas des interactions en groupe permettant de réunir plusieurs personnes dans le même espace d'échange.

11. : Il arrive que face à l'insistance des messages et la non disponibilité de l'interlocuteur d'avoir des interactions comme : « on parle après, je te réponds dès que possible, etc. » où tout simplement un «vu» affichant que le message est lu et marquant la non disponibilité du partenaire de l'échange.

12. : Nous avons demandé aux informateurs de créer un profil Facebook spécifique à l'étude pour ne pas dévoiler leurs identités cependant, certains ont gardé le même profil personnel. De ce fait, nous avons procédé, dans certains cas, à la suppression des éléments susceptibles de révéler l'identité de nos informateurs (photo de profil, nom de famille, informations personnelles, etc.

13. : Nous avons supprimé l'identifiant (pour garder l'anonymat) vu que la majorité des étudiants utilisent les noms de famille comme appellatifs au lieu des prénoms.

\section{RÉSUMÉS}

Notre contribution se veut une étude comparative de deux moyens d'échanges, l'interaction en ligne (IL) et l'interaction en présentiel (IP), en vue de dévoiler les particularités de ces deux formes d'échange. Le corpus composé des interactions quotidiennes et des interactions via Messenger (Facebook), nous a permis de comparer et contraster ces deux modes en se basant sur les points suivants : le cadre participatif, les rituels (ouverture et clôture), la langue parlée en interaction, la progression thématique, les attitudes et les émotions. Ainsi, les interactions en ligne se présentent comme un lieu d'échanges où les usagers adaptent leur comportement langagier et interactionnel en fonction des modes qu'offre cet espace numérique.

Our contribution is intended to be a comparative study of two means of exchange, online interaction (OI) and face-to-face interaction, (FI) in order to reveal the particularities of these two forms of exchange. The corpus composed of daily interactions and interactions via Messenger (Facebook), allowed us to compare and contrast these two modes based on the following points: the participatory framework, the rituals (opening and closing), the spoken language in interaction, thematic progression, attitudes and emotions. Thus, online interactions are presented as a place of exchange where users adapt their language and interactional behavior according to the modes offered by this digital space.

\section{INDEX}

Mots-clés : interaction en ligne, interaction en présentiel, cadre participatif, rituel d'ouverture et de clôture, progression thématique

Keywords : online interaction, face-to-face interaction, participatory framework, opening and closing rituals, thematic progression 
AUTEUR

KHEIRA YAHIAOUI

Maitresse de conférences HDR, Ecole Normale Supérieure-Oran, (Algérie) 